\title{
672 THE EFFECT OF CHEMORADIOTHERAPY AND TUMOR HISTOLOGY ON THE IMMUNE CONTEXTURE OF TUMOR- DRAINING LYMPH NODES IN NSCLC
}

${ }^{1}$ Marieke Fransen*, ${ }^{1}$ Famke Schneiders, ${ }^{1}$ Vinitha Kandiah, ${ }^{2}$ Teodora Radonic, ${ }^{2}$ Idris Bahce, ${ }^{2}$ Chris Dickhoff, ${ }^{1}$ Tanja de Gruijl. ${ }^{1}$ Cancer Center Amsterdam, Amsterdam UMC, Amsterdam, Netherlands; ${ }^{2}$ Amsterdam UMC, Amsterdam, Netherlands

Background Recently, the concept of locally delivered immune modulatory agents (re-)invigorating sub-optimally primed tumor-specific $\mathrm{T}$ cells and lifting suppression in the tumor microenvironment (TME) and tumor-draining lymph nodes (TDLN) has gained attention. TDLN play an important role in the induction of tumor-specific effector $\mathrm{T}$ cells. It is here that specialized dendritic cell (DC) subsets present tumorderived antigens to naïve $\mathrm{T}$ cells and start effective adaptive immune responses to cancer. Unfortunately, TDLN are also rapidly targeted by tumors for immune suppression, which may impair the efficacy of immunotherapy. Currently, there is limited knowledge on the immune contexture of TDLN in non-small cell lung cancer (NSCLC), differences between types of tumor histology, and the influence of standard treatment.

Methods In an exploratory study, we collected and analyzed viable cells from TDLN from patients with NSCLC, scheduled for surgical resection. To date, we have analyzed 43 TDLN from a total of 10 patients with multiparameter flowcytometry panels, either untreated or after neoadjuvant chemoradiotherapy (nCRT).

Results Our analyses reveal differences between squamous cell carcinoma (SCC) and adenocarcinoma (AC), discernable even within this small cohort. In AC, higher levels of PD-L1 on $\mathrm{CD} 11 \mathrm{c}+\mathrm{CD} 1 \mathrm{c}-\mathrm{LN}$-resident macrophages and $\mathrm{CD} 1 \mathrm{a}+$ migratory DC were accompanied by a lower activation state of CD8 + T cells by PD-1, CTLA-4 and CD69 expression levels. Furthermore, we found decreased activation of LN-resident DCs (by PD-L1 and CD83 levels) and a striking decrease in PD-1 and CD69 on CD8+ T cells, a decrease in effector and central memory CD8 $+\mathrm{T}$ cells, and an increase in naïve CD8 $+\mathrm{T}$ cells and Treg subsets after nCRT treatment, the current standard treatment of stage III NSCLC patients.

Conclusions These AC/SCC -related differences and nCRTinduced alterations in the immune status of hold clues for future patient stratification and combinatorial design of CRT with immunotherapy.

Ethics Approval This study was approved by the Medical Ethics Committee; 2017.545

http://dx.doi.org/10.1136/jitc-2021-SITC2021.672 